\title{
Modularization Method Based on New Layout Design in Conceptual Design Stage - Application of Multi-material Lightweight Structures Utilizing Machine Learning
}

\author{
Yasuo Asaga, Hidekazu Nishigaki \\ Toyota Central R\&D Labs.,Inc., Japan
}

\begin{abstract}
By creating a frame structure of a new layout and dividing it into modules with little coupling to each other, as well as using topology optimization and clustering, we have determined the assembly units of the product and the assembly process with least rework. Replacing materials with lightweight alternatives is an effective method for reducing the weight of structures. However, because adhesion and coefficients of thermal expansion are different for each material, it seems reasonable to replace modules represented as a functional unit. Therefore, we constructed a system for structural evaluation and material replacement with cross section that maintains equivalent stiffness for each module. We present the method of constructing the system and the effectiveness of using machine learning; confirmed by applying it to a box structure.
\end{abstract}

Keywords: Clustering, design structure matrix, frame structure, machine learning, modular design, multi-material, neural network, topology optimization

\section{Introduction}

To create new added value while improving manufacturing productivity in small quantities, it is suggested to make product variants using modules (Sebastian, 2015). We consider that it is effective to decompose a structure into modules as functional units for a wide variety of products, and to increase production efficiency by using modular assembly units. Additionally, weight reduction of structures is also important for reducing the environmental load of production. In weight reduction of mobility technologies, it is effective to replace different materials with well-designed modular lightweight alternatives. Design Structure Matrix (DSM) (Eppinger and Browning, 2012) analysis is considered an effective method for extracting module candidates. As an application of DSM to vehicles (Pandremenos and Chryssolouris, 2011), the relationship between 38 pressed parts of a vehicle body is described as a DSM, and 5 module decomposition candidates (of the front, floor and rear structures, as well as, lower and upper frames of a passenger compartment) are obtained by sorting the DSM using a self-organizing neural network method. In another study (Linden and Sellgren, 2016), the relationship between people and parts including the operation of a truck cab and bodily sensations, are described in the DSM. Technical problems are reduced by analyzing relations such as between customer demands and vibration transmission. In the application of these DSMs to vehicles, it is assumed that the components have functions and areas defined in advance, such as the geometric and force relationships (and requirements) between pressed parts. Furthermore, product part sizes are influenced by manufacturing methods and constraints 
Part IV: New Methods and Algorithms

(Boothroyd et al., 2010) (Lyu, et al., 2005). In other words, even if we modularize a conventional structure using DSM analysis, module candidates depend on part dimensions influenced and constrained by manufacturing processes. Therefore, we think that it is necessary to target an ideal new layout structure with no conventional manufacturing constraints during the conceptual design stage. For this reason, topology optimization (Bendsøe and Kikuchi, 1988; and Sigmund, 2003) which can calculate ideal new layout structures was implemented here. In previous study (Nishigaki and Asaga, 2017), we proposed a modularization method that utilized topology optimization for an ideal new layout structure, with DSM clustering and no conventional manufacturing constraints, to derive module candidates (see Figure 1). To obtain clear part dimensions, we adopt topology optimization using beam elements rather than solid elements; to not derive unclear boundaries. In the DSM analysis, we express the connection and physical characteristics of each beam element as a DSM. Using the dendrogram obtained after hierarchical clustering on the DSM, we determined the modular units and the assembly order of the product with least rework. Here, we calculated all the combinations of steelto-aluminum replacement on a modular unit, and examined the changes systematically with structural evaluations to extract an efficient substitution pattern for a lightweight structure. Because the cross-sectional size obtained by topology optimization is important physically in the structure, we add the polar moment of area to the DSM as a weight. Additionally, in hierarchical cluster analysis, we used cosine similarity (Wierzcho'n and Kłopotek, 2018) representing the closeness of the directions of the two vectors as an index of similarity between clusters; not Euclidean distance. Finally, after machine learning with a neural network using all these combination results, we show that it is possible to derive the material combinations for a lightweight structure with modular units that achieve specified performances.

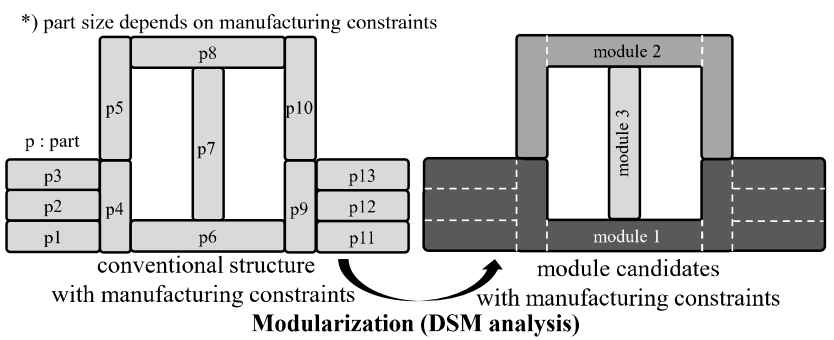

(a) Conventional method

*) part is a minimum unit of topology optimization

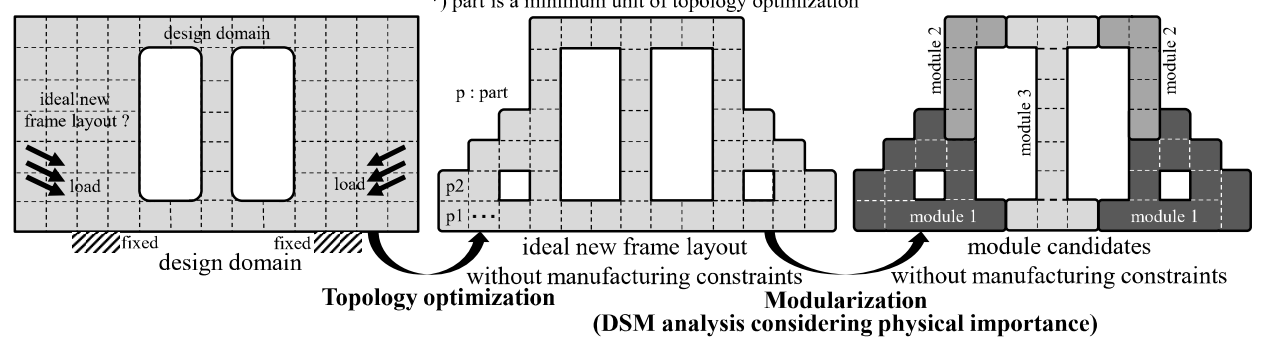

(b) Proposed method

Figure 1. Concept image of modularization method 


\section{Y. Asaga, H. Nishigaki}

\section{Methodology}

In the conceptual design stage, to easily estimate the mechanical performances of the multimaterial lightweight structure after material replacement, we have constructed a method that consists of four major steps.

(1) Topology optimization: Create an ideal new layout consisting of beam structure elements for the frame structure (called the ground structure), that maximizes stiffness with a total volume constraint.

(2) Modularization: Express the connection and member characteristics of each beam element as a DSM. Apply hierarchical clustering (Nielsen, 2016) to the DSM, extract candidate modules with the smallest coupling to others and express the assembly order of the product with least rework using a dendrogram. Here, the process images of step 1 and 2 is shown in Figure 2.

(3) Material replacement and characteristic analysis: Calculate the cross sectional characteristics with equivalent stiffness on a modular unit of each material replacement. Then, perform characteristic analysis (mass, height of the center of gravity and 1st eigenvalue) of the entire structure for all material combinations.

(4) Machine learning and prediction: Train the neural network with these results and predict the combination of material replacement on modular units satisfying the specified performances. Perform characteristic analysis with the predicted material combination to confirm performance achievement.

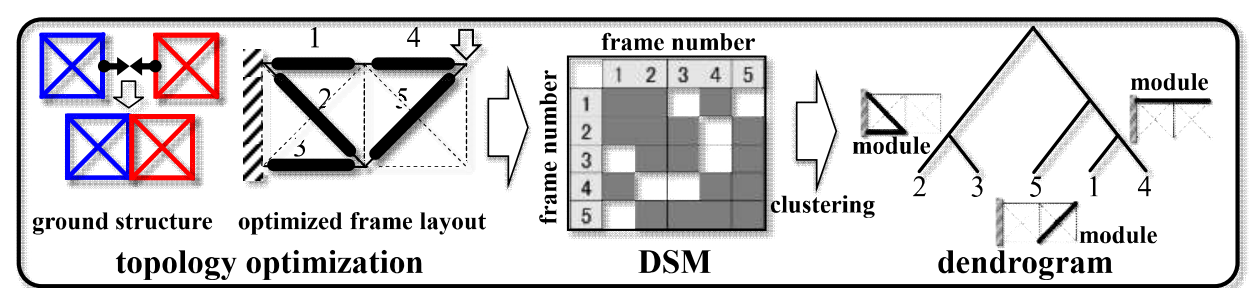

Figure 2. Process image of (1) topology optimization and (2) DSM analysis

\subsection{Topology optimization}

We apply topology optimization to create an ideal new layout of a frame structure consisting of many beam elements (Nishigaki et al., 2000). As a methodology to maximize the stiffness of the structure with a total volume constraint, we apply (a) a ground structure approach and (b) minimization of mean compliance (equivalent to stiffness maximization). To solve multi-loading conditions, we use the min-max approach which considers the loading condition that receives the maximum damage (mean compliance) at each iteration step. In the ground structure approach (see left of Figure 2), fixed nodes are preset, and beam elements are connected for all combinations between the box nodes. The design variable is the diameter of the hollow cylinder section, and the thickness is defined as the diameter multiplied by constant number. Then, the design variables are updated in proportion to the mean compliance as sensitivity. Resultantly, necessary elements become thicker and unnecessary ones become thinner. 
Part IV: New Methods and Algorithms

\subsection{Modularization method}

A DSM is constructed from the frame structure after topology optimization and module candidates are extracted by hierarchical clustering on the DSM (see center of Figure 2). Here, the beam elements of the frame layout obtained by topology optimization are regarded as parts, and the dependency between the beam elements is expressed by the DSM. However, if the DSM is composed of only connections between the parts, boundaries between module candidates can be physically weak. Therefore, considering that the cross-sectional size of the frame obtained by topology optimization is of high physical importance, the polar moment of area is thus added to the DSM as a weight. For example, in the case of a circular structure using the DSM composed of only connections between the parts, boundaries of module candidates equally exist on the arc. Thus, we cannot determine the unique module boundaries. Generally, we design physically important parts to be thicker in mechanical design. For this reason, we have introduced DSM analysis considering the polar moment of area with physical importance. In hierarchical cluster analysis, cosine similarity representing the closeness of the directions of the two vectors, is used a similarity index between clusters; not Euclidean distance representing the length of a line segment connecting two vectors. By using cosine similarity, it is possible to perform modularization taking into consideration the closeness and angles between parts. In other words, by focusing on the component ratio of the DSM element vector, similarity is evaluated by the physical importance of each element in connection with the overall structure. The group average method (Wierzcho'n and Kłopotek, 2018) is used as a method of defining the distance between clusters to be merged. By showing the connection relationship of clusters in a diagram called dendrogram, we can determine the modular units and the assembly order of the product with least rework (see right of Figure 2).

\subsection{Material replacement and characteristic analysis}

Replacing heavier material with a lightweight alternative is an effective method for reducing the weight of structures, but in order to minimize influences such as adhesiveness and differences in coefficients of thermal expansion due to the use of different materials, it seems reasonable to replace modules as functional units. Additionally, stiffness can decrease when replacing heavier materials with more lightweight alternatives. Therefore, we constructed a system for structural evaluation by replacing the characteristics of the material and the cross section with one that maintains equivalent stiffness for each module. Characteristic analysis (mass, height of the center of gravity and 1st eigenvalue) was then performed for all material combinations. In strength of materials, beam deflection is inversely proportional to EI, where, E is the Young's modulus and I is the second moment of area. In the case of a cantilever beam of length $\mathrm{L}$, the deflection $\delta$ when a load $\mathrm{P}$ is applied to the end point is given by $\delta=\mathrm{PL}^{3} /(3 \mathrm{EI})$. Therefore, if the index representing the state before material replacement is defined as " 1 ", and the index representing the state after material replacement is " 2 ", the following stiffness equation (1) should be satisfied to have the same stiffness. Here, topology optimization was performed on steel, and the lightweight material selected was aluminum. Here, the index 1 represents steel as the state before material replacement. The index 2 represents aluminum as the state after material replacement. In this topology optimization based on ground structure approach, the radius 


\section{Y. Asaga, H. Nishigaki}

$\mathrm{r}$ of the cylindrical cross section is used as a design variable, and the thickness $\mathrm{t}$ is a factor $\beta$ times the radius $r$ (see Figure 3). The second moment of area I of the thin cylindrical section is expressed by the following equation (2). Therefore, the radius $r_{2}$ of equivalent stiffness after replacement with a lightweight material is expressed by the following equation (3).

$$
\begin{aligned}
& E_{1} I_{1}=E_{2} I_{2} \\
& I=(\pi / 4)\left(r^{4}-(r-\beta r)^{4}\right)=(\pi / 4)\left(1-(1-\beta)^{4}\right) r^{4}(2) \\
& r_{2}=r_{I}\left(E_{1} / E_{2}\right)(1 / 4)
\end{aligned}
$$

Figure 3. Design variables of cylindrical cross section with equivalent stiffness

\subsection{Machine learning and prediction}

The neural network is trained using the results of the characteristic analysis (mass, height of the center of gravity and 1 st eigenvalue) of all combinations of material replacement based on equivalent stiffness on the modular unit. The number of learning times is set to 10,000 . The output values are a combination of material selection for each module. Using machine learning through the neural network, we can derive the combinations of material replacement on a modular unit satisfying the specified performances (mass, height of the center of gravity and 1 st eigenvalue) (see Figure 4).

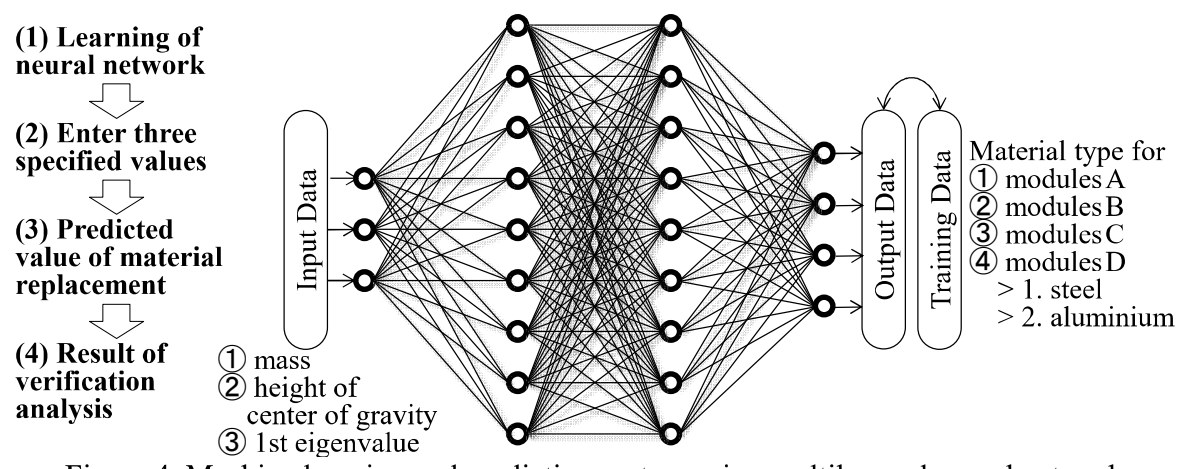

Figure 4. Machine learning and prediction system using multilayered neural network

We use multi-layer neural networks classified as mapping networks (the 4 layers consist of 3 neurons in the input layer, 9 neurons in the hidden layers and 4 neurons in the output layer) and backpropagation law (LeCun et al., 1998) based on minimizing the differences in the final output of the network. 


\section{Application of multi-material lightweight structures}

\subsection{Results of topology optimization}

We apply our methodology to a simple box structure and confirm its effectiveness. The ground structure consists of beam elements in the designable space and five load conditions are defined (see Figure 5). In this case, only rectangular elements are generated without diagonal elements in consideration of ease of manufacturing. Here, the maximum diameter of the beam element is set to 150 [mm], the volume constraint is set to $20 \%$ (final volume / volume when all beam elements have the maximum diameter), and the plate thickness $\mathrm{t}$ is set to 0.02 times the beam element radius. As a result of topology optimization (see Figure 6), it can be confirmed that the elements not contributing to the load path are eliminated or become thinner, and those contributing to the load path become thicker relatively, depending on the physical importance.

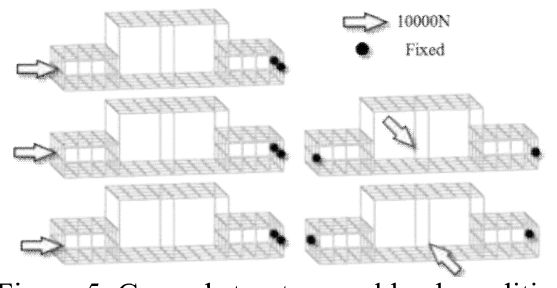

Figure 5. Ground structure and load conditions

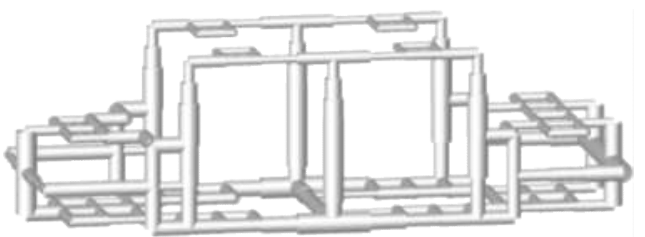

Figure 6. Result of topology optimization

\subsection{Results of modularization}

The beam elements forming the frame layout (see Figure 6) obtained by topology optimization are regarded as parts, and the dependency between the beam elements are expressed by the DSM (see left of Figure 7).
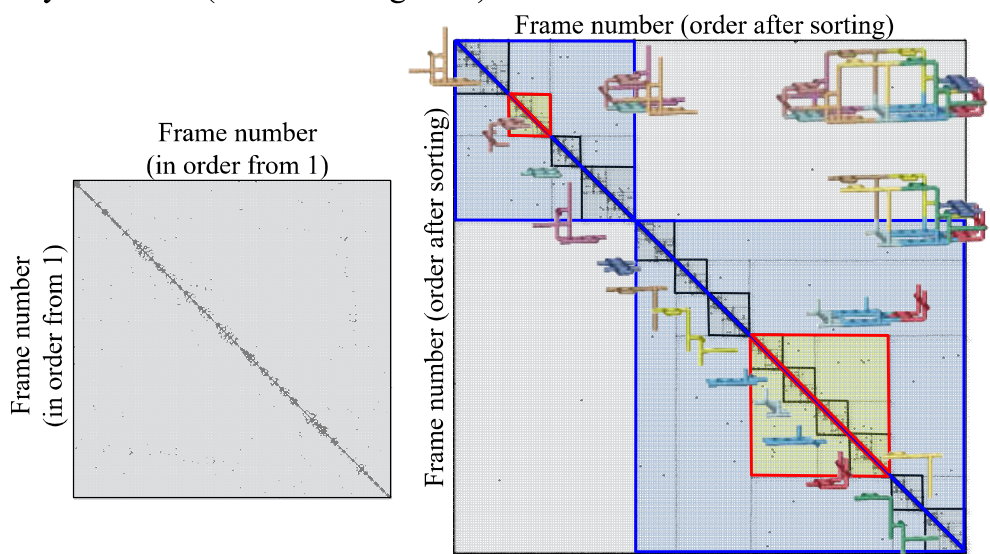

Figure 7. Design structure matrix (left: before clustering, right: after clustering)

Additionally, considering that the cross-sectional size of the frame is of high physical importance in the structure, we added the polar moment of area as a weight to the DSM. By hierarchical clustering, large and small square blocks were extracted as module 


\section{Y. Asaga, H. Nishigaki}

candidates with the smallest coupling to others (see right of Figure 7). Furthermore, the dendrogram shown in Figure 8 is obtained by hierarchical clustering. Then, the process of merging small triangles to form a large triangle can be considered as an efficient assembly order with least rework. Here, we defined four modules (A to D), as illustrated in Figure 8.

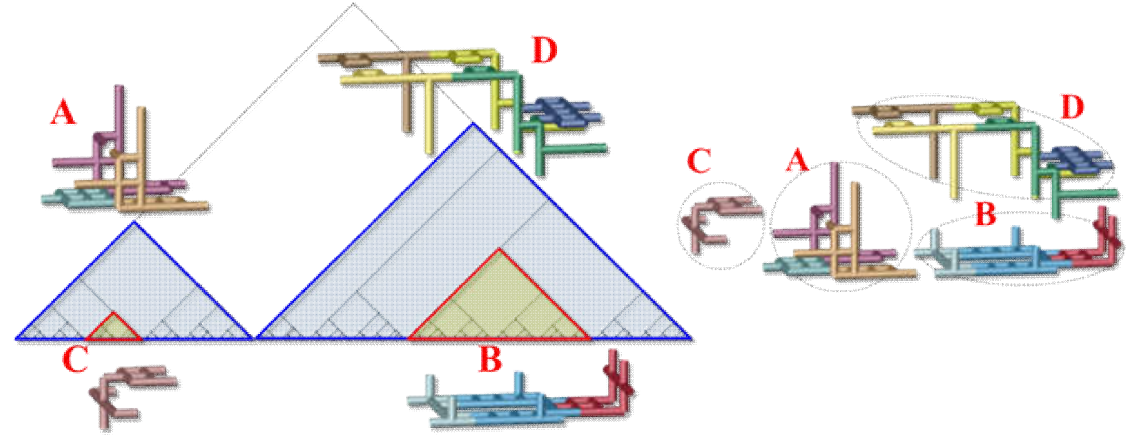

Figure 8. Dendrogram obtained by clustering

\subsection{Results of material replacement and characteristic analysis}

We show all the material combinations on a modular unit (see Figure 9) and the results of the characteristic analysis (see left of Figure 11) of all 16 cases of material replacement from steel to aluminum.

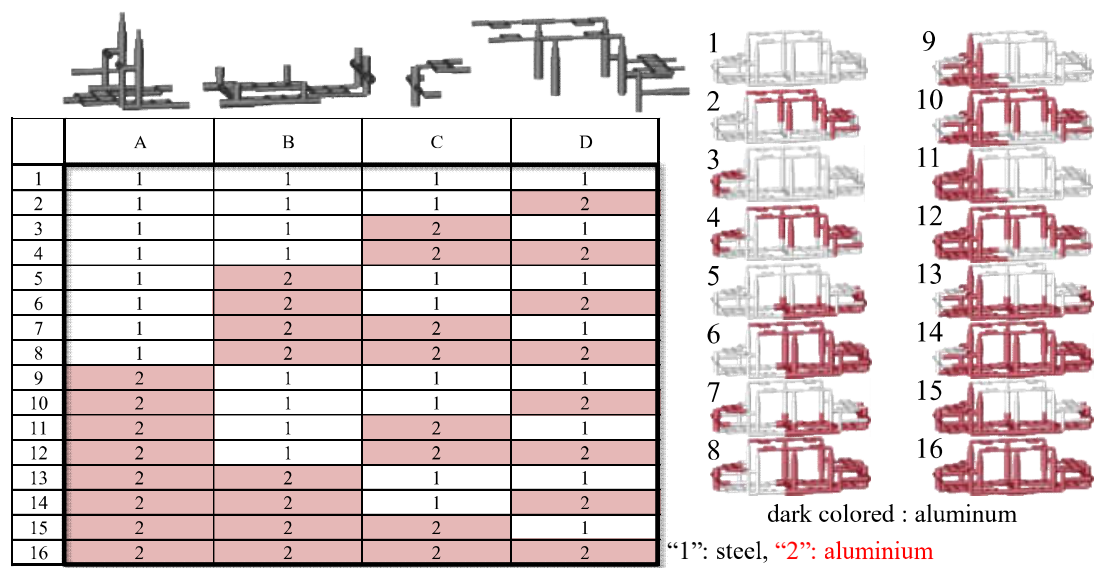

Figure 9. All combinations of replacing steel with lightweight aluminum

The height of the center of gravity changes significantly due to replacement of steel with lightweight materials (the height of the center of gravity is an important index for the stability of moving structures). 1st eigenvalues will be increased by decreasing the mass due to the lightweight alternatives with equivalent overall stiffness (see equation 3). Because of the material replacement on a modular unit, eigenmodes and antinodes of vibration may change (see Figure 10). Here, a maximum displacement error of $10.8 \%$ occurs on the basis of the displacement of case 1 using all steel. In this study, we proposed 
Part IV: New Methods and Algorithms

a method to realize equivalent stiffness by scaling the diameter (see equation 3 ) based on simple beam theory; assuming elasticity. However, as force and moments act on beam elements in complex structures, we found that it is necessary to extend the analysis to complex states, or to scale with a correction factor. However, as the purpose of this study is to extract the replacement regions and confirm their effectiveness in weight reduction and property improvements and displacement errors are relatively small, the issue regarding displacement errors is a subject for future research.

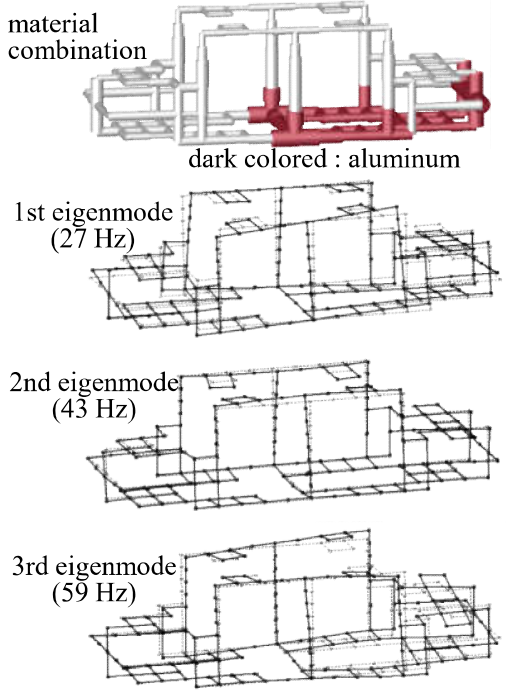

case 5

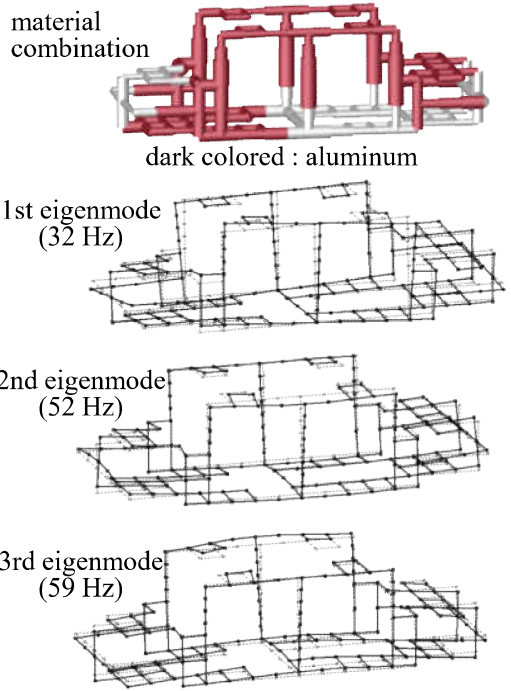

case 10

Figure 10. Examples of eigenmode of case 5 and case 10 combinations

\subsection{Results of machine learning and prediction}

We trained the neural network using all the results from the characteristic analysis and the material combinations (see Figure 11). Here, the 1st, 2nd and 3rd eigenvalues are important indices for the stability of a moving structure (see Figure 10). As one of the solution to handling multiple eigenvalues, we consider that additional neuron layers will be needed, like in deep learning. However, here we deal with only the 1st eigenvalue as an input value to the neural network for confirmations of the methodology. The handling of multiple eigenvalues in training a neural network will be the theme in future research. Resultantly, we have confirmed that the maximum error was as small as 0.028 and the model learned sufficiently well. Using this learned neural network, we have derived the combinations of material replacement on a modular unit realizing the specified values (the mass is reduced by $20 \%$ to 111.8 [kg], the height of the center of gravity is reduced by $40 \mathrm{~mm}$ to 30.5 [cm] and the 1 st eigenvalue is increased by $3 \mathrm{~Hz}$ to $29.6 \mathrm{~Hz}$ ) with respect to the result of case 1 in the initial state (steel only). Resultantly, module A is 1.0 , module B is 1.6 , module $\mathrm{C}$ is 1.0 and module $\mathrm{D}$ is 2.0 were obtained from the neural network. It was shown that module $\mathrm{A}$ and $\mathrm{C}$ should be steel, module D should be aluminum and module B should be mixed material (ratio 1.6) of steel and aluminum. One proposal for realizing this mixed material 


\section{Y. Asaga, H. Nishigaki}

state is to divide module B into smaller modular units (obtained by the above-described clustering) and to select a material for each of these units. The mixed material properties in verification analysis corresponding to 1.6 are determined by equations (4) and (5). Here, we defined the indices so that 1 is steel, 2 is aluminum, E is Young's modulus and $\rho$ is density. As the characteristic analysis using this material composition has errors within $3.7 \%$, we confirmed the effectiveness of our method. Finally, as we deal with steel and aluminum as a design variable like the binarization problem in this paper, there is an uncertainty problem. We consider that it will be effective to use logical approach or probability distribution from probability theory, as a design variable in the next step.

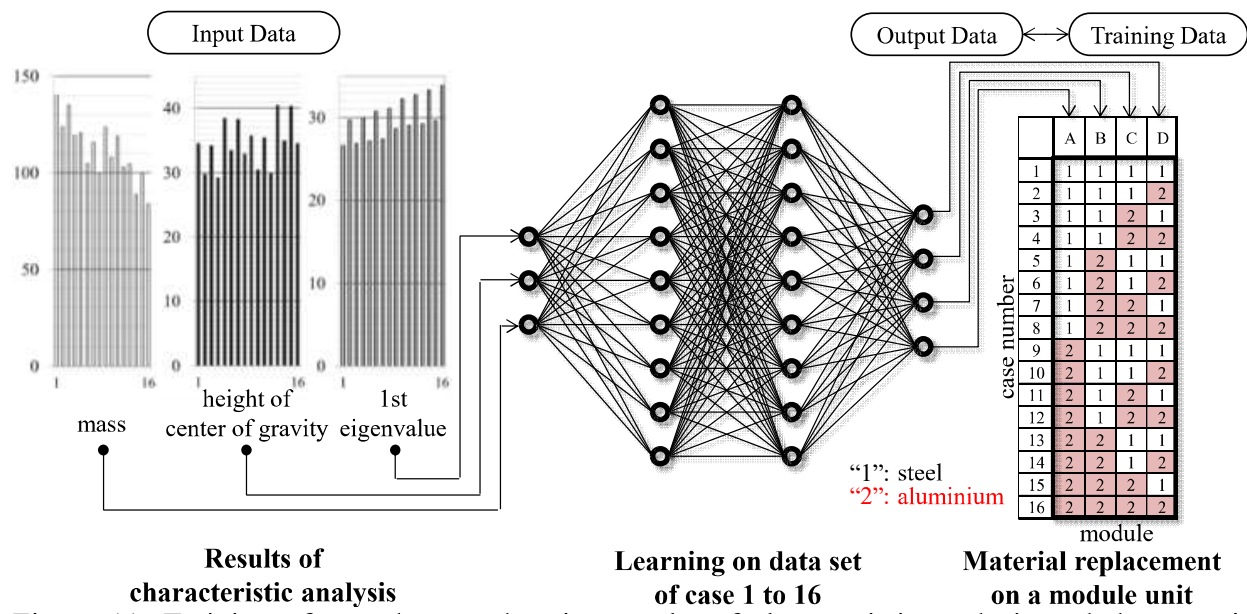

Figure 11. Training of neural network using results of characteristic analysis and the material combinations

$E(1.6)=E_{1}+\left(E_{2}-E_{1}\right)(1.6-1)$

$\rho(1.6)=\rho_{1}+\left(\rho_{2}-\rho_{1}\right)(1.6-1)$

\section{Conclusion}

Here, we propose a modularization method that utilizes topology optimization and clustering to derive module candidates and efficient assembly order, for an ideal new layout structure having no conventional manufacturing constraints. Here, we design a cross section with equivalent stiffness upon material replacement (steel-to-aluminum) to reduce the weight of the structure without reducing stiffness. Then, after machine learning with neural network using all the material combinations and their respective performances (mass, height of the center of gravity and 1st eigenvalue), we show that it is possible to derive combinations of replacement material on a modular unit that can achieve the specified performances. 
Part IV: New Methods and Algorithms

\section{References}

Bendsøe, M.P., Kikuchi, N., 1988. Generating Optimal Topologies in Structural Design Using a Homogenization Method, Computer Methods in Applied Mechanics and Engineering 71, pp.197-224.

Bendsøe, M.P., Sigmund, O., 2003. Topology Optimization : Theory, Methods, and Applications, Springer-Verlag.

Boothroyd, G., Dewhurst, P., Knight, W.A., 2010. Product Design for Manufacture and Assembly. Third Edition, CRC Press, pp.29-72,

Eppinger, S. D., Browning, T. R., 2012. Design structure matrix methods and applications, The MIT Press.

LeCun, Y., Bottou, L., Orr, G.B., Müller, K., 1998. Efficient BackProp, Neural Networks: tricks of the trade, Springer, pp.9-48.

Linden, J., Sellgren, U., Soderberg, A., 2016. Model-based reliability analysis, Artificial Intelligence for Engineering Design, Analysis and Manufacturing 30(3), pp.277-288.

Lyu, N., Lee, B., Saitou, K., 2005. Optimal subassembly partitioning of space frame structures for In-process dimensional adjustability and stiffness, Journal of Mechanical Design 128(3), pp.527-535.

Nielsen, F., 2016. Hierarchical clustering, Introduction to HPC with MPI for Data Science, Springer, pp.195-211.

Nishigaki, H., Asaga, Y., 2017, Modularization method based on creation of new layout structure, Transactions of the JSME (in Japanese), Vol. 83, No. 853 (2017), DOI:10.1299/transjsme.17-00065.

Nishigaki, H., Nishiwaki, S., Amago, T., Kikuchi, N., 2000. First order analysis for automotive body structure design, ASME DETC2000/DAC-14533.

Pandremenos, J., Chryssolouris, G., 2011. A neural network approach for the development of modular product architectures, International Journal of Computer Integrated Manufacturing 24(10), pp.879-887.

Sebastian, W., 2015. The Mobility revolution in the automotive industry: how not to miss the digital turnpike, Springer.

Wierzcho'n, S.T., Kłopotek, M.A., 2018. Modern algorithms of cluster analysis, Springer International Publishing AG.

Contact: Y. Asaga, Toyota Central R\&D Labs.,Inc., Material Mass Management Lab., 41-1, Yokomichi, Nagakute, Aichi, Japan, Phone: +81-0561-71-7451, Fax: +81-0561-63-6125, e-mail: asaga@mosk.tytlabs.co.jp, URL: https://www.tytlabs.co.jp/ 